\title{
Kinematics of the dwarf elliptical galaxy IC 225
}

\author{
Bryan W. Miller ${ }^{1}$ and Gwen Rudie ${ }^{2}$ \\ ${ }^{1}$ Gemini Observatory, \\ Casilla 603, La Serena, Chile \\ email: bmiller@gemini.edu \\ ${ }^{2}$ Astronomy Department, \\ California Institute of Technology, \\ Pasedena, CA 91125, USA
}

\begin{abstract}
We present the 2D kinematics of the nuclear region of the double-nucleated lowluminosity elliptical galaxy IC 225. Observations with the GMOS-N IFU at Gemini Observatory show a large amount of metal-rich gas coincident with the off-center nucleus. However, preliminary kinematics analysis indicates that the velocity of the stars in the off-center nucleus is distinct from that of the gas at the same position. Therefore, this is an intriguing example of how the formation of a nucleus or bulge may be occurring in a low-luminosity elliptical galaxy.
\end{abstract}

Keywords. galaxies: dwarf, galaxies: kinematics and dynamics, galaxies: nuclei

Nuclei are commonly found in the centers of late-type spiral and dwarf elliptical galaxies. These "central-massive objects" (CMO) fall on the same $M_{C M O}-M_{s p h}$ relation as super-massive black holes, suggesting a connection between the formation of nuclei and both black hole and spheroid or bulge growth (Wehner \& Harris 2006). However, it is uncertain how nuclei form. Two options are in-situ star formation and the merger of star clusters. Recently a bright $\mathrm{dE}$ has been discovered with a double nucleus and signs of on-going star formation ( $\mathrm{Gu}$ et al. 2006). With integral-field kinematic and stellar population information we may be able to identify the processes that are at work.

Observations of IC 225 were taken with the GMOS-N Integral Field Unit (IFU) in December 2005. This gave full spectral coverage $(\lambda=4200-5400 \AA)$ over a $5^{\prime \prime} \times 7^{\prime \prime}$ field with $0.2^{\prime \prime}$ sampling. The image quality was $0.5^{\prime \prime}$. The data were reduced using the Gemini IRAF package. For kinematic analysis the data cubes were binned to sufficient signal-to-noise using Voronoi adaptive 2D binning (Cappellari \& Copin 2003). The pPXF method of Cappellari \& Emsellem (2004) was used for the stellar kinematic analysis and to separate the stellar and gas components of the spectra. The IRAF task fxcor was then used to measure the emission line velocity field.

Figure 1 shows the morphology of the central region of IC 225 with the two nuclei and associated gas clouds. The source on the left in (a) is the central nucleus as it lies most near to the geometric center of the galaxy. The source on the right is the off-center nucleus. Most of the nebular emission appears coincident with the off-center nucleus.

However, the kinematics tells a different story (Figure 2). There is a suggestion of rotation in the gas about the vertical (E-W) axis. There are clouds of approaching gas on the south and east sides of the central nucleus. Also, there is a velocity reversal in the gas between the area just east of (below) the central nucleus and the off-center nucleus. However, the main stellar motion seen in the right panel is found in the stars close to the off-center nucleus. This reveals a stellar population that is kinematically distinct from the rest of the galaxy and from the gas at the same location. 


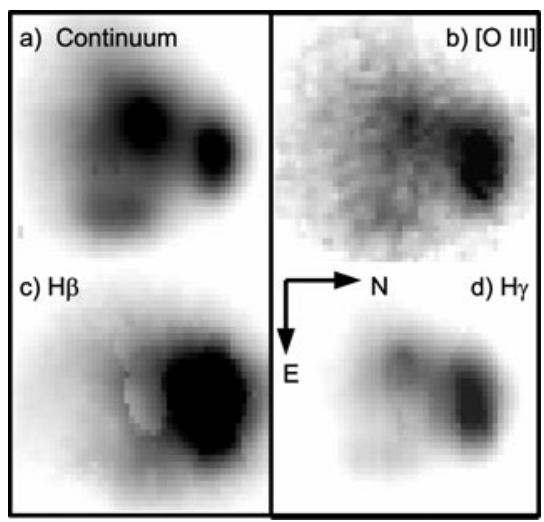

Figure 1. Narrow band images in a region of continuum (a) and three emission lines (b-d) reveal the presence of two distinct nuclei and the locations of clouds of gas.
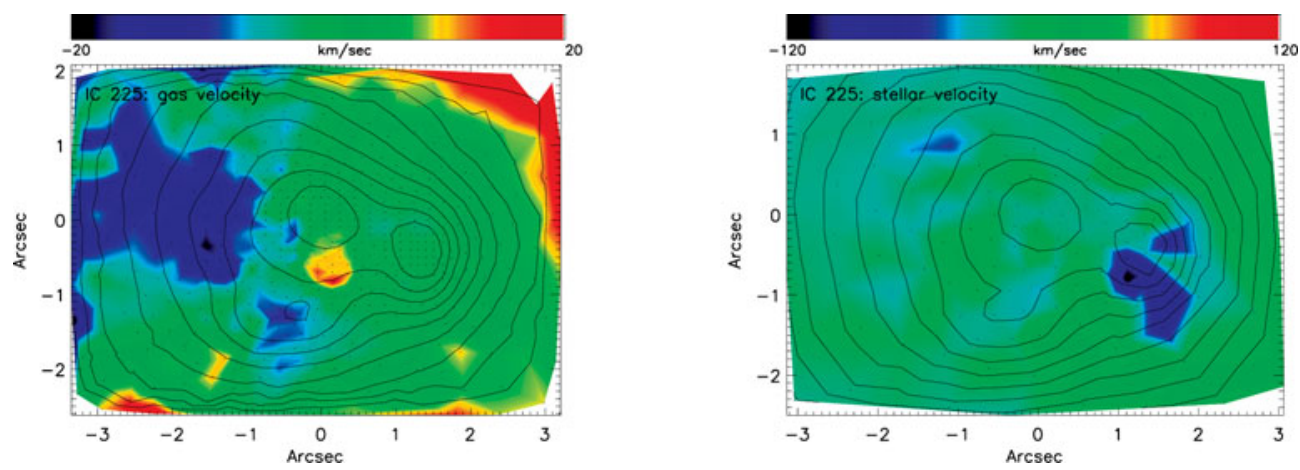

Figure 2. The left panel shows the relative velocity field of the gas within IC 225 as measured from the optical emission lines. The right panel gives the stellar velocity field. The contours show the continuum emission. One arcsecond corresponds to $100 \mathrm{pc}$ at the distance of $20 \mathrm{Mpc}$.

\section{Acknowledgements}

Based on observations obtained at the Gemini Observatory, which is operated by the Association of Universities for Research in Astronomy, Inc., under a cooperative agreement with the NSF on behalf of the Gemini partnership: the National Science Foundation (United States), the Particle Physics and Astronomy Research Council (United Kingdom), the National Research Council (Canada), CONICYT (Chile), the Australian Research Council (Australia), CNPq (Brazil), and CONICET (Argentina).

\section{References}

Cappellari, M. \& Copin, Y. 2003, MNRAS, 342, 345

Cappellari, M. \& Emsellem, E. 2004, PASP, 116, 138

Gu, Q., Zhao, Y., Shi, L., Peng, Z., \& Luo, X. 2006, AJ, 131, 806

Wehner, E. H. \& Harris, W. E. 2006, Ap. Lett., 644, L17 